\title{
Das Alter der Federmesser-Zivilisation auf Grund neuer naturwissenschaftlicher Untersuchungen
}

\author{
Von Hermann Schwabedissen, Köln
}

mit Beiträgen von Rudolf SchütrumpF, Kiel und Karl Otto Münnich, Heidelberg

Mit 6 Abbildungen im Text

$\mathrm{Z}$ us a m me $\mathrm{n}$ ass ung. Mit Hilfe verschiedener naturwissenschaftlicher Untersuchungsmethoden konnte die früher von uns als ausklingendes Magdalénien angesprochene FedermesserZivilisation des nordwesteuropäischen Flachlandes über die typologische Einstufung hinaus genau datiert werden.

Nach der Pollenanalyse gehören alle drei Gruppen dieser Zivilisation - die Rissener, Wehlener und Tjonger-Gruppe - in das späte Alleröd oder in den Übergang Alleröd / Jüngere Dryaszeit. Durch C14-Messungen wurde dies bestätigt. Danach liegt das Alter der drei Federmesser-Gruppen etwa zwischen 9500 und 9000 v. Chr. Geb.

$\mathrm{R}$ é $\mathrm{s}$ u mé . Au moyen de différentes méthodes scientifiques, la civilisation de "Federmesser" (dans la plaine du Nord-Ouest de l’Europe) qui a été caractérisée comme Magdalénien tardif, a pu être datée exactement au delà de la classification typologique.

Selon les résultats obtenus par l'analyse pollinique, les trois groupes de cette civilisation, ceux de Rissen, de Wehlen et de Tjonger, appartiennent tous à l'Alleröd finissant ou à l'époque transitoire entre l'Alleröd et le Dryas ultérieur. D'après ces analyses, l'origine des trois groupes de "Federmesser" est situé à peu près entre 9500 et 9000 avant J.-C.

Cela veut dire que la civilisation de "Federmesser" appartient encore au Pleistocène. A l'avenir il resterait à résoudre le problème de la date de l'Azilien par une analyse C14 pour obtenir la relation chronologique entre la civilisation de "Federmesser" et celle de l'Azilien.

\section{1.) Allgemeines, Fundverhältnisse und urgeschichtliche Bedeutung der naturwissenschaftlichen Ergebnisse (H. SchwabedisSEN)}

Eine große Zahl von Oberflächenfundplätzen hatte uns zur Herausstellung der „Federmesser-Zivilisation“ im nordwesteuropäischen Flachlande geführt $(12,14)$. Wir glaubten, diese in drei geographisch und formenkundlich verschiedene Untergruppen die Rissener, Wehlener und Tjonger-Gruppe - gliedern und als ausklingendes bzw. nachklingendes Magdalénien in das Spätglazial einordnen zu können (15). Zunächst lagen keine stratigraphischen Anhaltspunkte vor, so daß die kulturelle und zeitliche Einstufung nur auf typologischem Wege möglich war.

An einem günstigen Fundplatz in $\mathrm{R}$ is s e $\mathrm{n}$ b. Hamburg (Fundplatz 14/14a) wurde durch Grabung ein geschlossener Kulturhorizont der Federmesser-Zivilisation unter einer Schicht der Ahrensburger Kultur freigelegt (Abb. 1 u. Lit. 13, 15). Da die Ahrensburger Kultur in Stellmoor von R. SchüTRUMPF (10) pollenanalytisch in die jüngere Dryas-Zeit datiert worden ist, war damit über die Flinttypologie hinaus ein erster stratigraphischer und botanischer Hinweis auf das Alter der Federmesser-Kultur, zumindest der Rissener Gruppe, gegeben. Dank besonderer Aufmerksamkeit fand K. StüLCKen unweit der vorgenannten Stelle am Rande eines Bombentrichters ein Profil mit einer Gyttjaschicht in größerer Tiefe (Abb.2). Anscheinend war in einer Senke zwischen Dünen ein Wasserbecken entstanden, in dem sich Faulschlammabsätze mit periodischen Sandeinschwemmungen gebildet haben. Nach der Lage des Gyttjapaketes - z. T. in 3 Meter Tiefe und unter einem Bodenhorizont, an dessen Basis auf Fundplatz 14/14a die Ahrensburger Kulturschicht aufgetreten war - konnte erwartet werden, daß hier mit Hilfe des Faulschlamms eine genauere Datierung des Federmesser-Horizontes möglich sein würde. Der Profilaufbau ist aus Abb. $3 \mathrm{zu}$ ersehen. R. SCHINDler vom Museum für Hamburgische Geschichte unternahm als zuständiger 


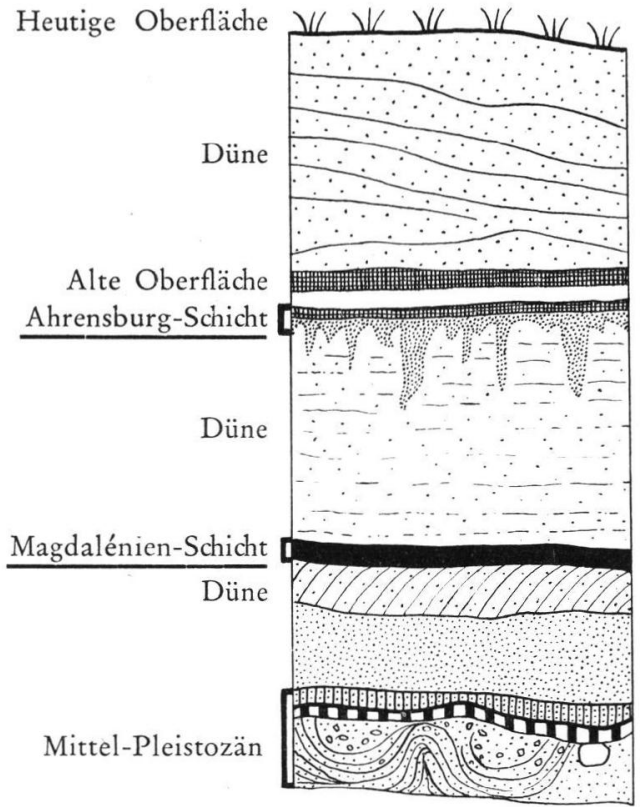

Abb. 1. Rissen, Fundplatz 14/14a

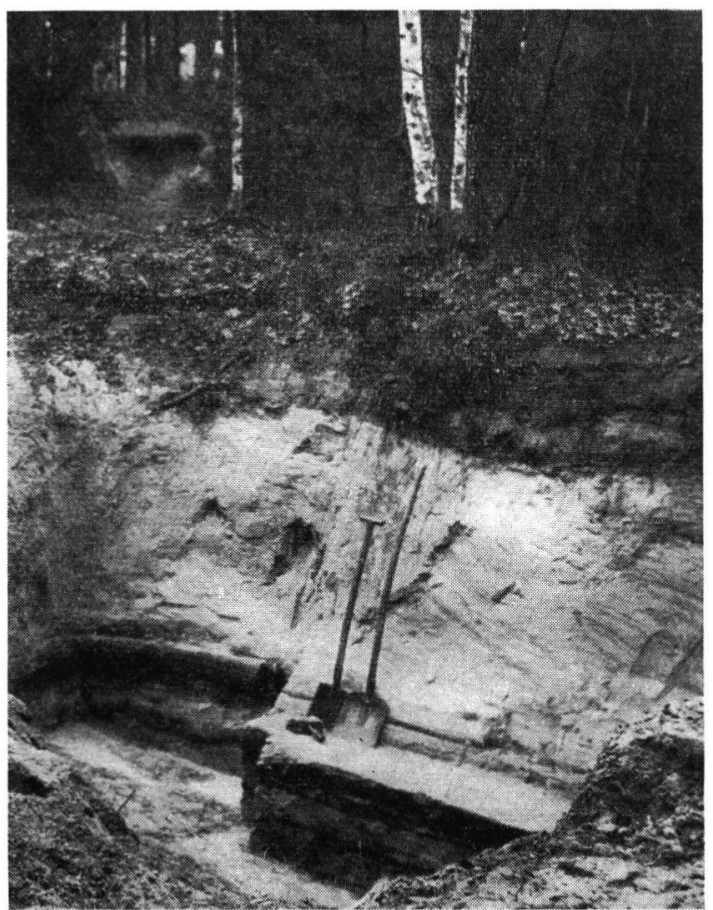

Abb. 2. Rissen-Bombentrichter

Gyttja = dunkle Schicht unter den Schaufeln (Foto K. Stülcken) 


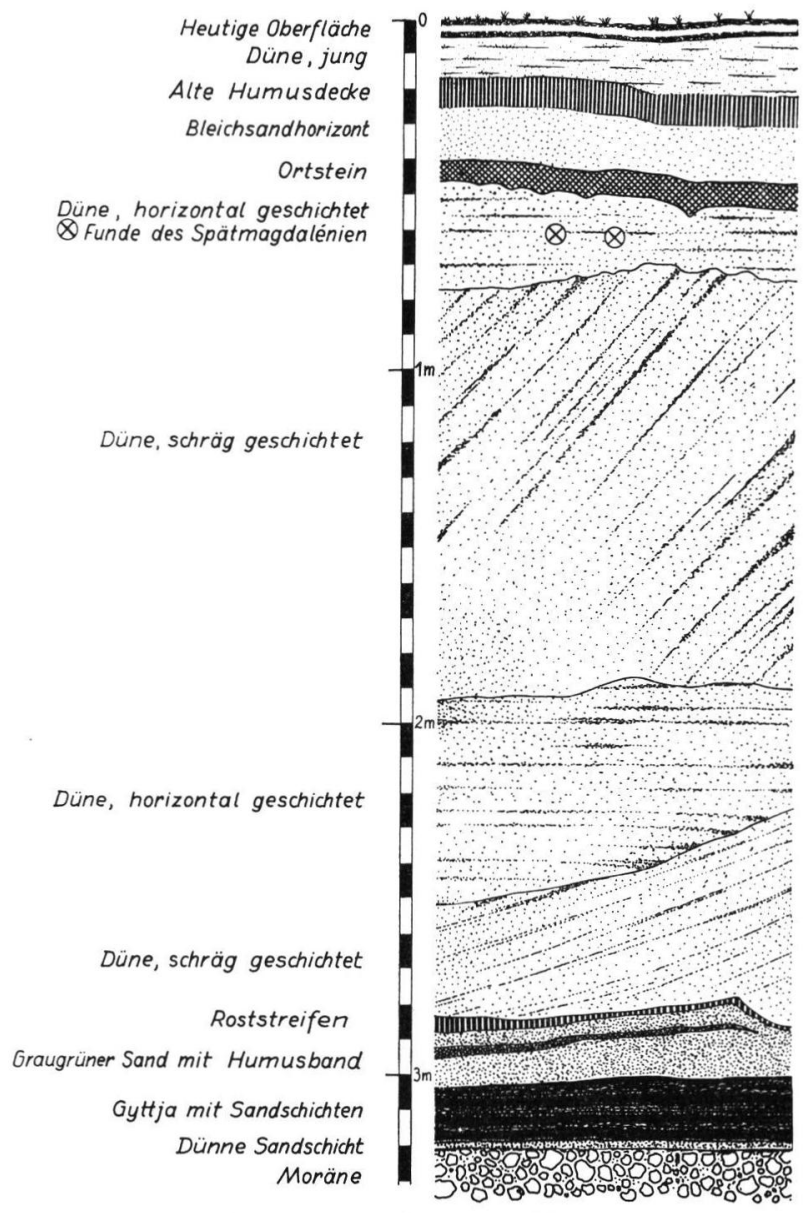

Abb. 3. Rissen-Bombentrichter

Denkmalpfleger in Absprache mit dem Altonaer Museum (Frl. M. PLAss) dankenswerterweise eine systematische Ausgrabung, bei der ein zusammenhängendes Profil hergestellt und ein größerer Teil der Gyttja untersucht wurde. Die Faulschlammschicht ergab keine Kulturüberreste. Im Profil traten einzelne Flintwerkzeuge auf, die zwar nicht auf einen geschlossenen Fundhorizont, aber doch auf den oberen Teil über der Gyttja lokalisiert werden konnten.

Die Faulschlammschichten wurden von R. SchütrumpF pollenanalytisch untersucht und in die Alleröd-Zeit datiert (vgl. Beitrag SchütrumpF). Wie ein Vergleich mit dem Profil an Fundplatz 14/14a und die allgemeine Fundstreuung nahegelegten, mußte die Federmesser-Kultur wahrscheinlich jünger sein als die Gyttja. Um den Fundhorizont genauer zu lokalisieren, wurde - ermutigt durch das Untersuchungsresultat am Fundplatz Rissen 14/14a von F. DürR (Abb. 4) - eine Schwermineralanalyse dieses Profils durch R. A. Hintz im Vergleich mit dem von Fundplatz 14/14a, das den Ahrensburger und Federmesser-Horizont enthielt und einem dritten Profil vom gleichen Aufbau in ca. 300 Meter Entfernung vorgenommen. Die Kurven der drei Profile zeigten den gleichen Rhythmus der Schwermineralsedimentation und ließen die Federmesser-Schicht über der Gyttja hervortreten. 
In jüngster Zeit wurde an der Wand des Bombentrichters ein Profilteil sichtbar, in dem sich die Kulturschicht, wie am Fundplatz 14/14a, als mit Holzkohle durchsetzte Schicht, über der Gyttja liegend, zu erkennen gab (Abb.5). Damit ist die Lage der Federmesser-Kultur über dem Faulschlamm und ihr jüngeres Alter als gesichert anzusehen.

Darüberhinaus hat K. O. MüNNICH vom II. Physikalischen Institut der Universität Heidelberg (vgl. Beitrag MüNnich) folgende Resultate von $\mathrm{C}^{14}-\mathrm{Messungen} \mathrm{erzielt:}$

Holz, unmittelbar unter der Gyttja (Rissen-Bombentrichter) $9980 \pm 290$ v. Chr. Geb.

Gyttja, unterer Teil (Rissen-Bombentrichter) $9600 \pm 280$ v. Chr. Geb.

Holzkohle aus der Federmesser-Schicht

(Fundplatz Rissen 14/14a)

$9500 \pm 180$ v. Chr. Geb.

Diese Ergebnisse besagen, daß 1.) die Gyttja auch nach der C14-Datierung älter ist als die Federmesser-Kultur und 2.) sowohl die Zahlen für Gyttja und Holz als auch die Zahl für die Kulturschicht nach den zahlreichen bekannten $\mathrm{C}^{14}$-Daten innerhalb des Alleröd liegen. Damit steht auch eine im $C^{14}$-Labor zu Kopenhagen (1) gemessene Zahl von $8350 \pm 350$ v. Chr. Geb. für die Jüngere Dryas-Zeit, der die das Spätmagdalénien von Rissen 14/14a überlagernde Ahrensburger Kultur angehört, im Einklang.

Nimmt man die Resultate von Pollenanalyse und Schwermineralanalyse ${ }^{1}$ ) hinzu, so muß die Federmesser-Kultur von Rissen sehr wahrscheinlich in einen späten Abschnitt der Allerödschwankung bis höchstens in den Übergang Alleröd/Jüngere DryasZeit fallen. Die Rissener Gruppe der Federmesser-Zivilisation kann damit am namengebenden Fundplatz als datiert gelten.

Sie wird erhärtet durch einen Befund von Lieth b. Elmshorn, Kr. Pinneberg. Hier konnte E. Kolumbe (Hamburg-Altona) ein Dünenprofil feststellen, in dem sich über einer Torfschicht ein klassisches Federmesser ${ }^{2}$ ) fand. Kolumbe's Pollenanalyse verweist den Torf ins Alleröd, dessen $C^{14}$-Alter $9270 \pm 350$ v. Chr. Geb. beträgt (C ${ }^{14}$-Messung New Haven Y-442). In Verbindung mit der Stratigraphie ist auch hier ein Hinweis dafür gegeben, daß die Federmesserzivilisation ans Ende oder in die Zeit kurz nach dem Alleröd gehört.

A. Rust (9) konnte bei B or n e ck ein Zeltfundament mit zahlreichen Werkzeugen unserer Wehlener Gruppe der Federmesser-Zivilisation freilegen. Einige offenbar zugehörige Fundstücke in der Gyttja des angrenzenden Ahrensburg-Meiendorfer Tunneltales wurden durch R. SchütrumpF (11) pollenanalytisch datiert und in den Übergang von der Allerödschwankung zur Jüngeren Dryaszeit gesetzt. Mithin scheint die Wehlener Gruppe der Federmesser-Zivilisation, trotz gewisser typologischer Besonderheiten, etwa der gleichen Zeit wie die Rissener Gruppe anzugehören.

In Us s e 1 o bei Enschede in Holland hat C. C. W. J. Heuszzelen (6, 7, 7a) kürzlich einen charakteristischen Fundplatz der Tjonger-Gruppe sorgfältig untersucht. Flintsachen und Holzkohle der im Sand gelegenen Fundschicht gehen in eine angrenzende, vermoorte Wasserrinne über. Die im Torf gelegene Holzkohle gehört nach der Pollenanalyse durch vaN DER HAMMEN $(2,5)$ in den Übergang Alleröd/Jüngere Dryas-Zeit. Es ist jedoch nicht ausgeschlossen, daß die Holzkohle erst nach der Zeit der Besiedlung

1) Bemerkenswert sind die Kurven der Ätzwerte für Granat und Hornblende bei F. DürR (Abb.4). Sie zeigen beide für die Postglazialzeit und für das Alleröd geringe Werte, für die Jüngere Dryaszeit dagegen ein zusammenhängendes Maximum. Wenn die Ätzwerte tatsächlich in ursächlicher Relation zu den Klimaphasen stehen, wie es scheint, so fiele die FedermesserSchicht von Rissen in den Ubergang Alleröd/Jüngere Dryaszeit. Sollten sich die Ergebnisse der Schwermineralanalyse wie im Sinne von Rissen weiter bestätigen, so hätten wir damit eine für manchen Fall brauchbare Datierungsmethode. Die Untersuchungen sollen wegen ihrer grundsätzlichen Bedeutung fortgesetzt werden.

2) Die Publikation von E. Kolumbe ist in Vorbereitung. 


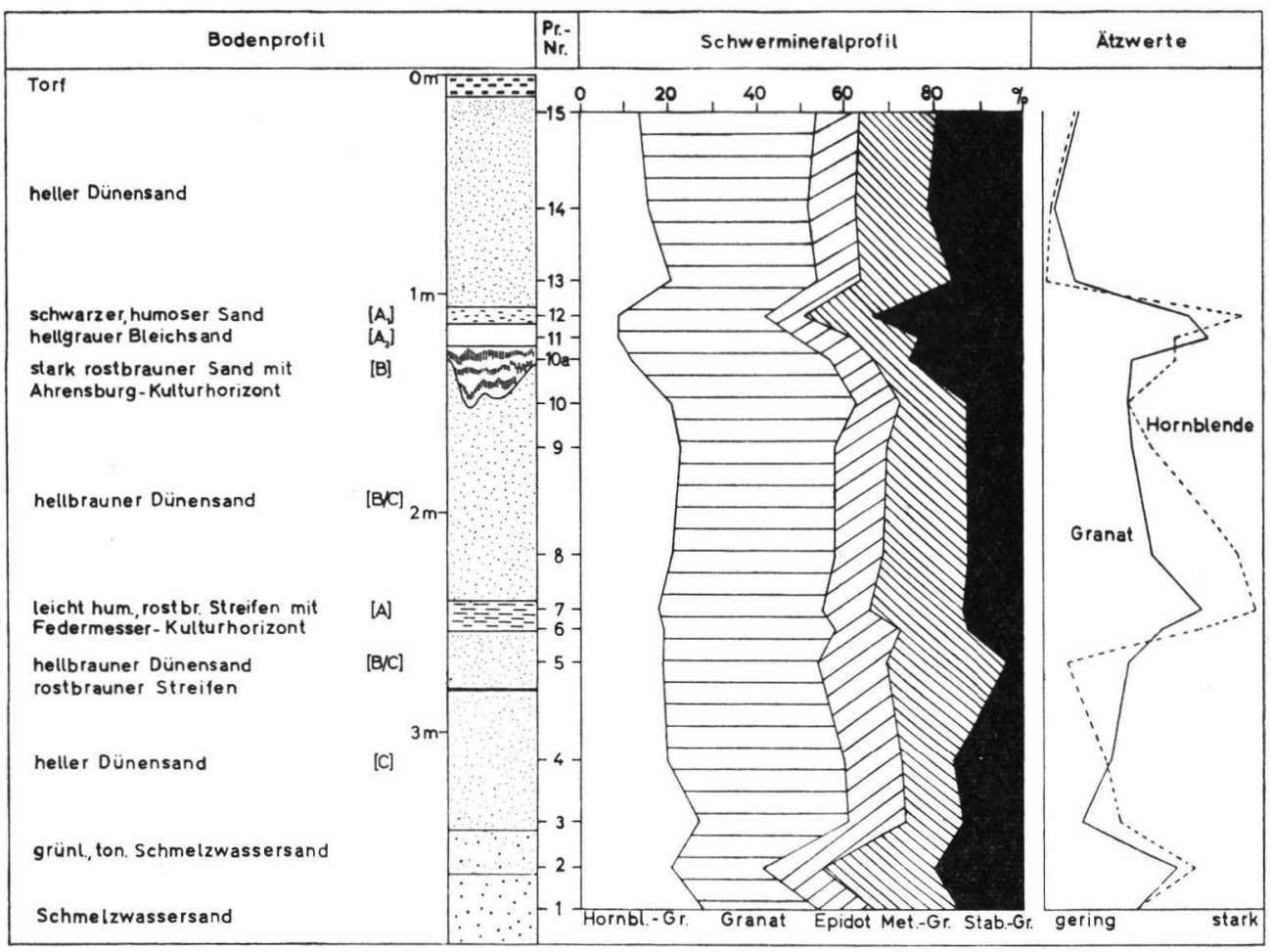

Abb. 4. Rissen Fpl. 14/14a - Schwermineralanalyse n. F. DürR

in die vermoorte Wasserrinne eingespült wurde. Deshalb datiert H. T. WATERBolK (16) die Fundschicht von Usselo allgemein in eine fortgeschrittene Phase des Allerc̈d, aber vor die eigentliche Jüngere Dryaszeit. Eine durch DE VRIEs in Groningen für die Holzkohle gewonnene $\mathrm{C}^{14}$-Zahl ist $9105 \pm 120$ v. Chr. Geb. Dieses Datum kann das richtige Alter für Usselo oder zumindest einen terminus ante quem bedeuten. Mit der $\mathrm{C}^{14}-\mathrm{Zahl}$ $9605 \pm 100$ v. Chr. Geb. (Gro 921) für den Hypnum-Torf im liegenden Sand ist ein terminus post quem gegeben ${ }^{3}$ ). Die Tjonger-Gruppe fällt also gleichfalls in das Ende der Alleröd-Schwankung oder in den Übergang zur Jüngeren Dryaszeit.

Damit kommen wir zu der Feststellung, daß alle drei Gruppen der FedermesserZivilisation des nordwesteuropäischen Flachlandes - die Rissener, die Wehlener und die Tjonger-Gruppe - auf Grund verschiedener naturwissenschaftlicher Untersuchungsmethoden in etwa die gleiche Zeit fallen. Das bedeutet eine Bestätigung der früher auf typologischem Wege gewonnenen Datierung der Federmesser-Zivilisation.

Für die Verknüpfung der Federmesser-Gruppen mit dem Spätmagdalénien standen uns auf den Oberflächenfundplätzen nur Steingerättypen zur Verfügung. Stationen wie die am Martinsberg b. Andernach ermöglichten mit ihrem Knochen- und Geweihgerät, vor allem den Harpunen, einen Anschluß an das echte Magdalénien Mittel- und Westeuropas. Auf Grund der Hinweise durch die teilweise datierte Tuffüberdeckung des Lagerplatzes am Martinsberg und die Faunenvergesellschaftung glaubten wir die Station zwischen dem Ende der Altesten Dryaszeit und dem mittleren Alleröd ein-

3) Die Mitteilung der Zahlen verdanke ich Herrn Kollegen WaterbolK; das Meßresultat wird demnächst in einem Bericht von Hl. DE Vries und H. T. WaterbolK in "Science" erscheinen. 


\title{
RISSEN-BOMBENTRICHTER
}

\author{
OSTTEIL / NORDWAND
}

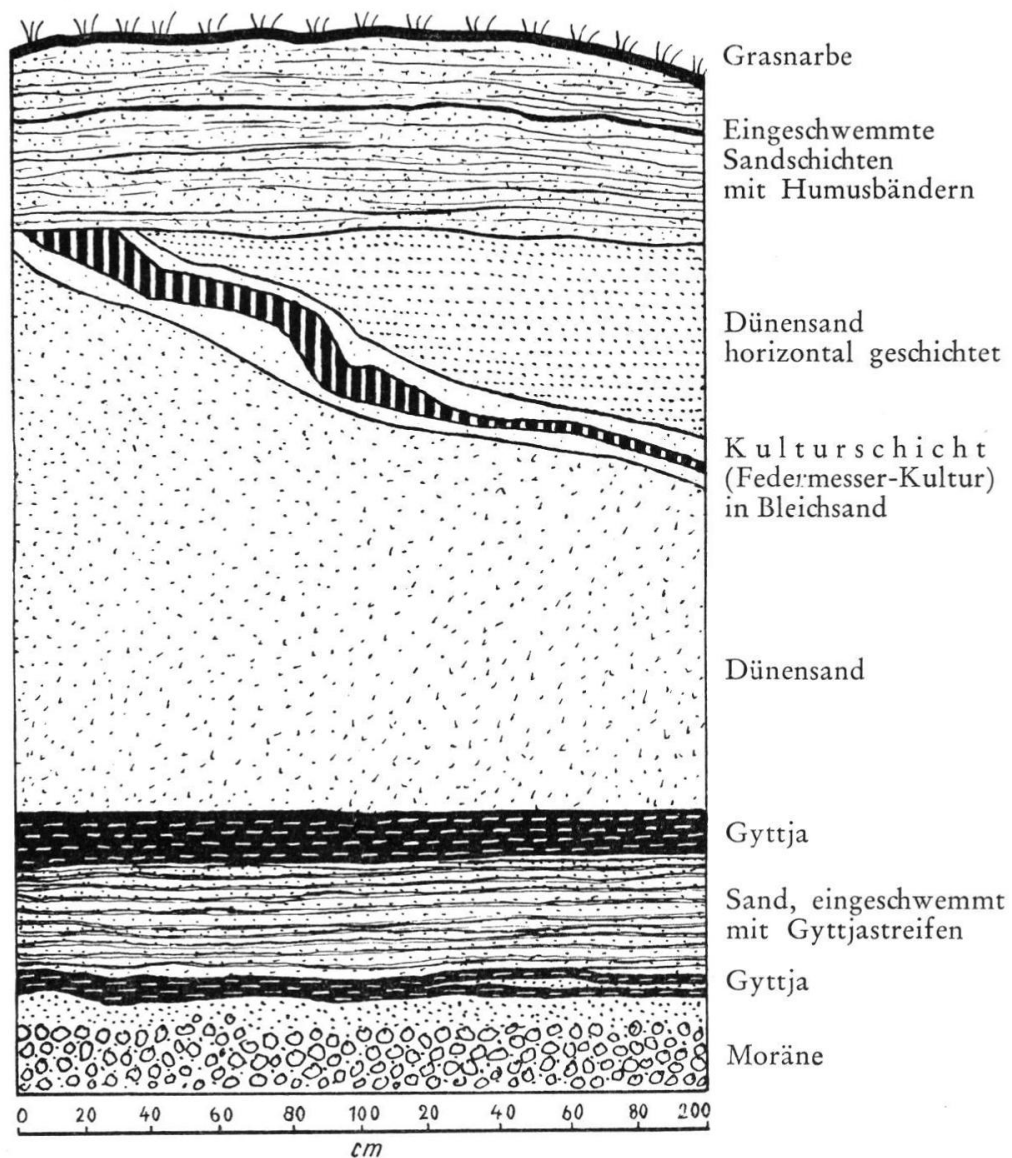

Abb. 5. Rissen-Bombentrichter, Ostteil / Nordwand

ordnen zu können. Mittlerweile haben die $\mathrm{C}^{14}$-Messungen in Heidelberg (vgl. Beitrag K. O. MünNich) für das Magdalénien vom Martinsberg ein Alter von $9350 \pm 220 \mathrm{v}$. Chr. Geb. (Geweih) ergeben. Da zahlreiche Kontrollmessungen gezeigt haben, daß C14-Datierungen von alten Geweihproben sehr häufig zu junge Ergebnisse liefern, kann daher das Spätmagdalénien vom Charakter der Martinsberg-Siedlung sehr wohl etwas älter sein als die Federmesser-Fundplätze im nordwesteuropäischen Flachlande.

In diese Richtung deuten auch die $\mathrm{C}^{14}$-Daten für die den Andernacher Fundplatz überdeckenden Bimssandschichten. Letztere wurden bekanntlich in W a 11 ensen im Hils im mittleren Alleröd wiedergefunden (4) und dort mit einem Alter von 9950 v. Chr. Geb. ( $\mathrm{H} 1 / 8)$ bzw. 9850 v. Chr. Geb. ( $\mathrm{H}$ 1/48) gleichfalls in Heidelberg gemessen. Andere $C^{14}$-Resultate für W a 11 ensen liegen mit $9210 \pm 320$ v. Chr. Geb. (K-107) und $9094 \pm 500$ v. Chr. Geb. (C-337) niedriger. Sämtliche Zahlen gehören jedoch in die für das Alleröd gemessene Spanne. Auch das französische Spätmagdalénien scheint in die Alleröd-Zeit zu fallen. Nach freundlicher Mitteilung von Herrn Gross (vgl. Aufsatz Gross in vorliegendem Band dieses Jahrbuches) hat die Datierung einer Holz- 
kohleprobe aus einer Kulturschicht des Magdalénien V/VI in einer Höhle von L a V a c h e b. Niaux (Ausgrabung R. Robert) $9700 \pm 200$ v. Chr. Geb. (L-336 C) ergeben. Die Station von Andernach (etwa Magdalénien VIa) dürfte keineswegs älter sein als die von $\mathrm{L}$ a $\mathrm{V}$ a che, eher jünger.

Im übrigen kann nach den vorliegenden Daten der Streit, ob die Funde von Andernach in primärem oder verschwemmtem Löß gelegen haben, als entschieden angesehen werden. Wenn das Magdalénien V/VI ins Alleröd fällt, wird kaum noch mit Lößbildung zu rechnen sein. Die alte Annahme, daß der Lagerplatz am $\mathrm{M}$ a r t ins be r g b. Andernach kurz vor der Tuffüberdeckung, d. h. also während oder etwas vor der mittleren Alleröd-Zeit bestanden hat, dürfte nicht nur den Fundverhältnissen am besten gerecht werden (15), sondern auch den C14-Daten entsprechen.

Was nun das Alterverhältnis des echten Spätmagdalénien und der Federmessergruppen betrifft, so will es scheinen als ob ersteres ein wenig älter sei. Die Datierungsergebnisse für die Federmesser-Stationen deuten ja, wie wir sahen, mehr auf das Ende des Alleröd oder auf den Übergang zur jüngeren Dryaszeit. Allerdings bleiben noch weitere $\mathrm{C}^{14}$-Messungen für das klassische Spätmagdalénien abzuwarten.

Auf keinen Fall ist das Magdalénien V/VI jünger als die Federmesser-Gruppen. Damit sind jetzt auch die chronologischen Voraussetzungen dafür gegeben, die Federmesser-Zivilisation von Stationen des Spätmagdalénien, wie Andernach oder anderen in Südwestdeutschland bzw. überhaupt des Westens, abzuleiten.

Der Hamburger Kultur gegenüber erweist sich die Federmesser-Zivilisation als jünger. Ein später Horizont der von A. Rust in Poggenwisch ausgegrabenen Hamburger Kultur gehört vegetationsgeschichtlich nach R. SchütrumpF (11) kurz vor die Bölling-Schwankung. Die zuverlässigste Messung - nämlich einer Holzprobe von Poggenwisch in Heidelberg ergab die C14-Zahl $11030 \pm 370$ v. Chr. Geb. (H 136/ 116). Damit stimmen die für das Bölling gewonnenen $C^{14}$-Daten gut überein.

Durch die mit Hilfe verschiedener naturwissenschaftlicher Methoden gewonnenen chronologischen Unterlagen wird mithin auch die Annahme erhärtet, daß seit der klimatisch günstigeren Allerödzeit Träger des ausklingenden Magdalénien von verschiedenen Gegenden aus in das nordwesteuropäische Flachland eingedrungen sind und zur Besiedlung jener Gebiete wesentlich beigetragen haben.

Als weitere Aufgabe ist nun die genauere Datierung des spätesten Magdalénien in Frankreich und des Azilien ins Auge zu fassen, um das zeitliche Verhältnis und die möglichen siedlungsgeschichtlichen Beziehungen dieser Fundgruppen zu der nordwesteuropäischen Federmesser-Zivilisation zu klären.

\section{Literatur}

1.) Anderson, E. C. / Levi, H. / Tauber, H.: Copenhagen Natural Radiocarbon Measurements, I. - Science 118, S. 6-11, 1953.

2.) Bohmers, A.: Jong-Palaeolithicum en Vroeg-Mesolithicum. - Gedenkbloek A. E. van Giffen, Meppel 1947.

3.) DürR, F.: Sedimentspetrographische Untersuchungen an einem Dünenpodsolprofil. - Schriften naturw. Ver. f. Schl.-Holst. 26/1, 1952.

4.) Firbas, F.: Die Vegetationsentwicklung im Spätglazial von Wallensen im Hils. - Nachr. Akad. Wiss. Göttingen, Biol.-physik.-chemische Abtl. Nr. 5, 1954.

5.) van der Hammen, T.: Late glacial flora und periglacial phenomena in the Netherlands. Leidse Geol. Med. 17, 1951.

6.) Heijszeler, C. C. W. J. / Florschütz, F.: Botanisch-archaeologisch oderzoek in Twente. Hand. Nat. en Gen. Congr. 1941.

7 ) Heijszeler, C. C. W. J.: De oudheidkundige opgravingen in Twente in de laatste jaren. Gedenkboek van Giffen, Meppel 1947.

7a.) - De leag van Usselo. - Grondboor en Hamer, Nr. 2, Dez. 1955.

8.) Iversen, J.: Radiocarbon Dating of the Alleröd Period. - Science 118, S. 6-11, 1953. 
9.) Rust, A.: Jungpaläolithische Wohnanlagen bei Hamburg. - Hammaburg 1, 1948.

10.) Schütrumpf, R.: Die pollenanalytische Untersuchung der Rentierjägerfundstätte Stellmoor. - In: Rust, A., Stellmoor, Neumünster 1943.

11.) - Das Spätglazial. - Eiszeitalter und Gegenwart 6, S. 41-51, 1955.

12.) Schwabedissen, H.: Die mittlere Steinzeit im westlichen Norddeutschland. - Neumünster 1944.

13.) - Hamburg-Rissen ein wichtiger Fundplatz der frühen Menschheitsgeschichte. - Hamimaburg 2, 1949.

14.) - Das Vorkommen des Magdalénien im nordwesteuropäischen Flachland. - Eiszeitalter und Gegenwart 1, S. 152-165, 1951.

15.) - Die Federmesser-Gruppen des nordwesteuropäischen Flachlandes. - Neumünster 1954.

16.) Waterbolk, H. T.: De Praehistoriske Mens en zijn Milieu. - Assen 1954.

\section{2.) Die Pollenanalytische Untersuchung der Gyttja-Schicht vom Fundplatz Rissen - Bombentrichter (R. SchütrumpF)}

In den bei der Ausgrabung des Rissener Steinzeit-Fundplatzes „Bombentrichter“ freigelegten Profilen zeigten sich in den Sanden unterhalb der paläolithischen Kulturschichten mehrere schmale, dunkel gefärbte Bänder, welche die Hoffnung aufkommen ließen, daß ihr Alter pollenanalytisch bestimmt werden könnte. Entsprechende Untersuchungen dieser Horizonte blieben zunächst jedoch ohne Erfolg. Es zeigte sich, daß die Dunkelfärbung nur zum geringsten Teil auf humose Bestandteile zurückzuführen ist. Es handelt sich vielmehr um minerogenes Material, das bei der Bodenbildung in dünnen, dunklen Lagen angereichert worden ist. Die Schichten waren daher zum größten Teil absolut pollenfrei. Nur in drei Fällen war der Humusgehalt etwas größer, und es war möglich, wenige Pollen darin zu finden. Die Pollenfrequenz ( 9 bis 15 pro $18 \times 18 \mathrm{~mm}$-Präparat nach Flußsäure-Aufschluß) war jedoch für eine quantitative Auszählung zu gering. Es wurden folgende Arten in nachstehendem absoluten Mengenverhältnis festgestellt:

Probe 1

Probe 2

Probe 3

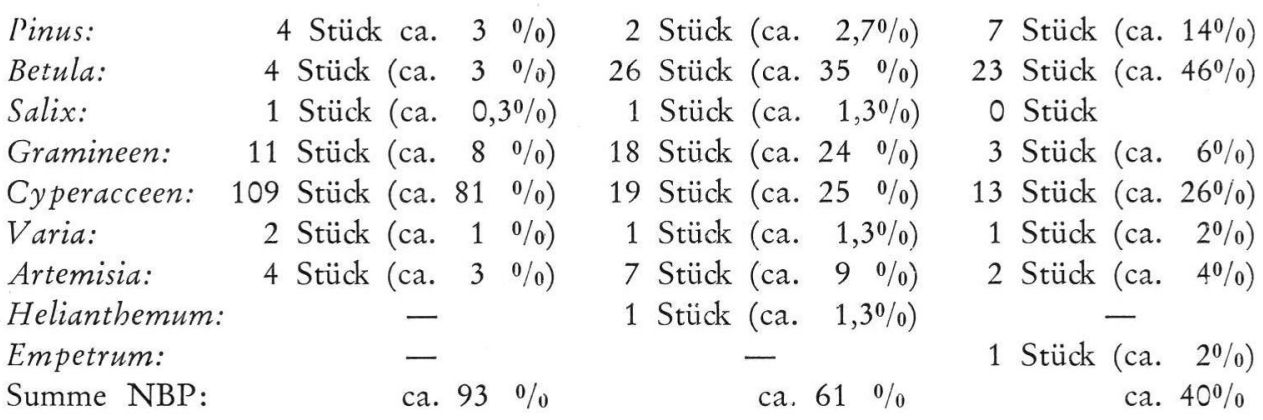

Nach der stratigraphischen Lage ist Probe Nr. 1 die älteste und Probe Nr. 3 die jüngste. Alle 3 Proben sind älter als die Funde. Nach der Artenzusammensetzung gehören alle Proben ins Spätglazial, wofür auch die verhältnismäßig hohe Nichtbaumpollensumme spricht. Eine Entscheidung darüber, $\mathrm{zu}$ welchem Unterabschnitt (Zone) des Spätglazials die Schichten im Einzelnen gehören, ermöglicht das Analysenergebnis aber nicht. Stratigraphisch sind sie älter als die Ahrensburger Stufe, da die entsprechende Kulturschicht im Profil höher liegt.

Im vergangenen Jahr wurde aus der näheren Umgebung der Fundstelle ein Profil bekannt, bei dem zwischen die Sandschichten eine sandige Gyttja eingeschaltet ist. Das 


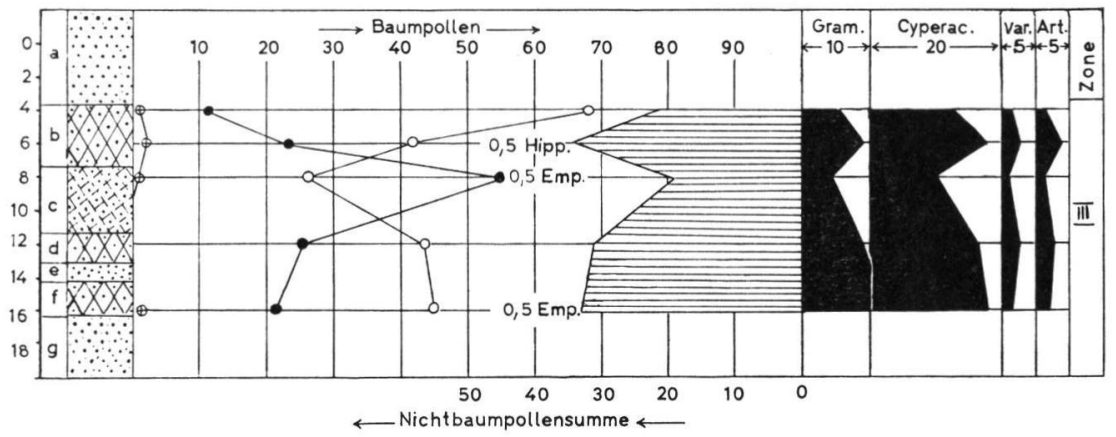

Abb. 6. Rissen-Bombentrichter, Pollendiagramm

Material wurde mir in Form eines $20 \mathrm{~cm}$ mächtigen Blockes auf Veranlassung von H. Schwabedissen von Fräulein M. Plass, Hamburg-Altona, zur Untersuchung zugesandt. Der Profilausschnitt hat folgenden Schicht-Aufbau:

$0-4 \mathrm{~cm}$ gelblich-weißer Feinsand (a)

$4-7 \mathrm{~cm}$ feinsandige Gyttja, blättrig spaltend, olivfarbig (b)

7-11 cm Gyttja-haltiger Feinsand, nicht aufblätternd, etwas heller (c)

11-16 cm feinsandige Gyttja, blättrig aufspaltend, olivfarbig $(\mathrm{d}+\mathrm{f})$; zwischen 13 und $14 \mathrm{~cm}$ eine reine Feinsand-Zwischenlage (e) (gelber Flugsand)

$16-20 \mathrm{~cm}$ gelber Feinsand (g).

$\mathrm{Da}$ Pollendichte und Pollenerhaltung in den untersuchten Gyttja-Proben normal waren, konnte ein kleines Diagramm (Abb. 6) aufgestellt werden. Es zeigt eindeutig, daß die gesamte Gyttjenfolge ebenso wie die oben beschriebenen Einzelproben ins Spätglazial gehören. Die Birke dominiert mit Ausnahme einer Unterbrechung durch ein Kiefern-Maximum von $55 \%$ in Probe 8 . Wir haben somit eine Sukzession BirkeKiefer-Birke, wie sie aus der Alleröd-Zeit bekanntgeworden ist. Die Weide erscheint nur sporadisch.

Nach diesem Befund können alle analysierten Spektren ins Alleröd, d.h. in die Pollenzone III, gestellt werden. Mit dieser chronologischen Einordnung stehen die hohe Pollenfrequenz (ca. 200 je $18 \times 18 \mathrm{~mm}$ ), die relativ geringe Nichtbaumpollensumme und die niedrigen Werte der für das Spätglazial charakteristischen Arten Artemisia, Hippophae und Empetrum in gutem Einklang.

Für die Zeitansetzung der Rissener Kulturschicht aus der „Federmesser-Gruppe“ ergibt sich durch die Altersstellung der Gyttja eine untere Grenze. Sie kann danach nur gleichaltrig oder jünger sein als ein später Abschnitt von Alleröd. $\mathrm{Da}$ in einem anderen Profil Funde der Ahrensburger Stufe über der Kulturschicht von Rissen liegen (vgl. Abb. 1), kann die Federmesser-Schicht eingeengt werden zwischen Alleröd und der Ahrensburger Stufe. Somit ergibt sich für die bisher aus Schleswig-Holstein bekannten spätpaläolithischen Kulturstufen auf Grund pollenanalytischer Untersuchungen etwa folgende chronologische Abfolge:

Meiendorf (ältere Hamburger Kultur)

Poggenwisch (jüngere Hamburger Kultur)

Rissen (Federmesser-Kultur)

Borneck (Federmesser-Kultur)

Stellmoor (Ahrensburger Kultur)
Alteste Dryaszeit Zone I

Alteste Dryaszeit Zone I

Alleröd (jüngeres?) Zone III

Ubergang

Zone III/IV

Jüngere Dryaszeit Zone IV 


\section{3.) Zur $\mathbf{C}^{14}$-Datierung der Federmesserkultur (K. O. MüNNICH)}

$$
\text { Probe }
$$

$\mathrm{C}^{14}$-Alter

\section{A) $\mathrm{R}$ is se $\mathrm{n}$}

H 21/18

Rissen. Gyttja aus einem Horizont unter der Kulturschicht, karbonatfrei, Kohlenstoffgehalt ca. $9 \%$

H 18/11 Rissen. Holz aus der Gyttja

$11550 \pm 280$ Jahre $\left.^{4}\right)$

$11930 \pm 290$

$11740 \pm 200$

H 75/68 Rissen. Verkohltes Holz aus der Kulturschicht

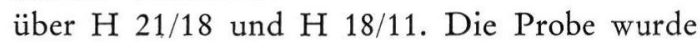
vor der Verbrennung durch Kochen in verdünnter Salzsäure gereinigt

$11450 \pm 180$

B) Martinsberg

H 85/91 Magdalénien vom Martinsberg bei Andernach. Geweih. Karbonat durch Salzsäure entfernt, Eiweißsubstanz durch Dialyse gereinigt und zur $C^{14}$-Analyse verbrannt

$11300 \pm 220$

Erfahrungsgemäß ergibt die $C^{14}$-Analyse von Knochen- und Geweihmaterial häufig ein niedrigeres Alter, als der archäologischen Einstufung entspricht, und zwar auch dann, wenn der in Knochen und Geweih enthaltene Kalk als von vornherein suspekt entfernt und nur die Eiweißsubstanz zur Analyse verwendet wird. Dies scheint daran zu liegen, daß Eiweißsubstanzen einer Verunreinigung durch jüngeres Material, etwa durch Humussäuren, die das Wasser im Boden mit sich führt, in höherem Maße unterliegen als die Zellulose des Holzes. Vor allem aber läßt sich der so eingedrungene fremde Kohlenstoff bei den Eiweißstoffen nur schwer entfernen, während dies bei Zellulose weit weniger Schwierigkeit bereitet. Meist tritt die Verschiebung des $\mathrm{C}^{14}$ Alters zu niedrigeren Werten bei der Eiweißsubstanz zusammen mit einer viel stärkeren gleichsinnigen Verfälschung des $\mathrm{C}^{14}$-Alters des Karbonats auf5).

Es ist demnach nicht unwahrscheinlich, daß das Alter der Magdalénien-Station am Martinsberg in Wirklichkeit um mehrere hundert Jahre höher ist, als es sich aus unserer $\mathrm{C}^{14}$-Analyse ergab.

Anschrift der Verf.: Prof. Dr. H. Schwabedissen, Inst. f. Ur- u. Frühgeschichte der Universität Köln, Weyertal 125.

Dr. R. Schütrumpf, Geol. Inst. u. Museum der Universität Kiel.

Dr. K. O. Münnich, II. Physik. Inst. der Universität Heidelberg.

Manuskript eingeg. 7. 3. 1957.

4) „mittlerer Fehler": Der angegebene Fehler ergibt sich aus dem mittleren statistischen Fehler der Zählung der radioaktiv zcrfallenden $\mathrm{C}^{14}$-Atome. Falls man voraussetzen darf, daß andere Fehler (z. B. Verunreinigung der Probe) vernachlässigt werden können, liegt das wirkliche Alter mit einer Wahrscheinlichkeit von $68 \%$ innerhalb des einfachen, mit einer Wahrscheinlichkeit von $95 \%$ innerhalb des doppelten und mit einer Wahrscheinlichkeit von $99,7 \%$ innerhalb des dreifachen mittleren Fehlers. Vgl. K. O. MünNICH, Heidelberg Radiocarbon Measurements I, Seience (im Druck).

5) vgl. K. O. MüNnich, Der C ${ }^{14-G e h a l t ~ v o n ~ G r u n d w a s s e r . ~ N a t u r w i s s . ~ 44, ~ 32 . ~} 1957$. 